\title{
Intraband absorption for normal illumination in quantum dot intermediate band solar cells
}

\author{
Antonio Luque*, Antonio Marti, Elisa Antolin, Pablo Garcia-Linares \\ instituto de Energía Solar, Universidad Politécnica de Madrid, 28040 Madrid, Spain
}

A R T I C L E I N F O

Keywords:

Solar cell

Intermediate band

Quantum dots

Quantum calculations

\begin{abstract}
A B S T R A C T
$\mathrm{n}$ the current intermediate band solar cells made with InAs quantum dots (QDS) in GaAs, the ransitions by absorption of photons between the intermediate band and the conduction band for Ilumination normal to the cell surface is very weak or, more often, undetectable. We model the $Q D$ as a jarallelepiped potential well and calculate the envelope function of the electron wavefunctions. By sbtaining the dipolar matrix elements we find that, with the present shapes, this absorption is . orbidden or very weak. Deeper QDs with smaller base dimensions should be made to permit this absorption.
\end{abstract}

\section{Introduction}

The intermediate band (IB) solar cell was proposed [1] to increase the efficiency of solar cells. In an IB solar cell, three absorption bands can produce electron-hole pair generation: the $V B \rightarrow C B$ (VB, valence band; CB, conduction band) transitions (the only one in single gap cells, i.e. cells unmodified with quantum dots) and two successive $V B \rightarrow I B$ and $I B \rightarrow C B$ transitions. $A$ very high detailed balance efficiency limit of about $63 \%$ versus $41 \%$ for a single junction solar cell was calculated.

Prototype cells have been manufactured by several groups using lnAs quantum dots (QDs) in a GaAs matrix [2-6] with a top efficiency of over $18 \%$ [6]. However, thermal and tunnel escape, rather than a second photon, are responsible for the $\mathrm{IB} \rightarrow \mathrm{CB}$ transition, also called intraband transition (because in these QDs the IB is detached from the $C B$ ) and, if this happens, the current will be increased but at the expense of a lower voltage. Two photons are necessary for thermodynamic reasons if the voltage is to be higher than the quantum efficiency (QE) threshold [7].

The choice of QDs instead of quantum wells (QWs) is in part to avoid the continuum of states represented by travelling functions in the $(x, y)$ plane that might provide an easy thermalization of the $\mathrm{JB}$ and the CB. But further to this, it is known that the $\mathrm{BB} \rightarrow \mathrm{CB}$ transition is forbidden (see for instance [8], p. 155) in QWs for vertical photons (not so for photons travelling in the $(x, y)$ plane). That is why the quantum dot infrared detectors (QDIDs) [9| came in as an attempt to substitute the QWID.

\footnotetext{
* Corresponding author.

E-mail address: a.Juque
}

In our QD IB cells, two-photon quantum efficiency (QE) experiments have been carried out to verify experimentally the desired mechanism [10] that, although present (in several batches differing in $\mathrm{QD}$ size, composition, etc.), has been found to be very weak, about three orders of magnitude below the $\mathrm{VB} \rightarrow \mathrm{IB}$, and only measurable at temperatures below $80 \mathrm{~K}$ (when thermal escape is reduced). The purpose of this paper is to determine whether there is some fundamental reason that prevents the desired optical [B $\rightarrow$ CB transitions. There might indeed be a technological reason: the IB is not sufficiently doped as to permit a partial filling of the $\mathrm{lB}-\mathrm{a}$ condition [1] necessary for the optical IB $\rightarrow$ CB transition-but even in cases of proper doping (determined by capacitive experiments) the two-photon current is small or undetectable.

\section{Eigenfunctions and eigenvalues}

In the $\boldsymbol{k} \cdot \boldsymbol{p}$ approximation the wavefunctions of the electrons in presence of a $Q D$ are described by an envelope function that modulates the periodic part $u_{0}(\boldsymbol{r})$ of the material Bloch function (for $k=0$ ) that carries the atomic details. In the case of an intraband transition the one-band approximation is adequate [11]; at least for qualitative and semiquantitative analyses. The envelope function is the eigenfunction of the following Schrödinger equation:

$-\frac{\hbar^{2}}{2 m} \nabla^{2} \varphi+V(\mathbf{r}) \varphi=E \varphi$

where $m$ is the effective mass of the electrons in the $C B$ and $V(\mathbf{r})$ is the minimum of the $\mathrm{CB}$. 
The purpose of this paper is to get a qualitative insight so that the mathematics will be simplified as much as possible. Thus, in this equation we are considering a scalar effective mass (spherical CB minimum) constant everywhere (we take the geometrical mean of the effective masses of the dot and barrier materials) and a square potential well whose height is the offset $U$ of the CB bottom in the $\mathrm{QD}$ and host materials. The strain effects and piezoelectric potentials that are very important in a quantitative analysis are disregarded here. The shape of the low potential region (the $Q D$ ) is usually admitted to be a strongly truncated [12], squat, quadrangular pyramid but we shall use a shallow squared box of dimensions $2 a, 2 b$, and $2 c$.

Later we shall justify the use of the separation-of-variables method. Thus, we set $\psi(x, y, z)=\alpha(x) \beta(y) \gamma(z)$. Each one-dimensional function must then be the solution of a one-dimensional Schrödinger equation with a symmetric one-dimensional potential well. This is a simple problem that can be found in any quantum mechanics textbook (for the one-dimensional case). We describe it here to set the nomenclature. In the case of $\gamma(z)$ the potential will be 0 for $|z|<c$ and $U$ outside this region. A similar discussion can be made for the $\alpha(x)$ and $\beta(y)$ functions. For $\gamma(z)$ the confined one-dimensional solutions inside the $(-c, c)$ interval will

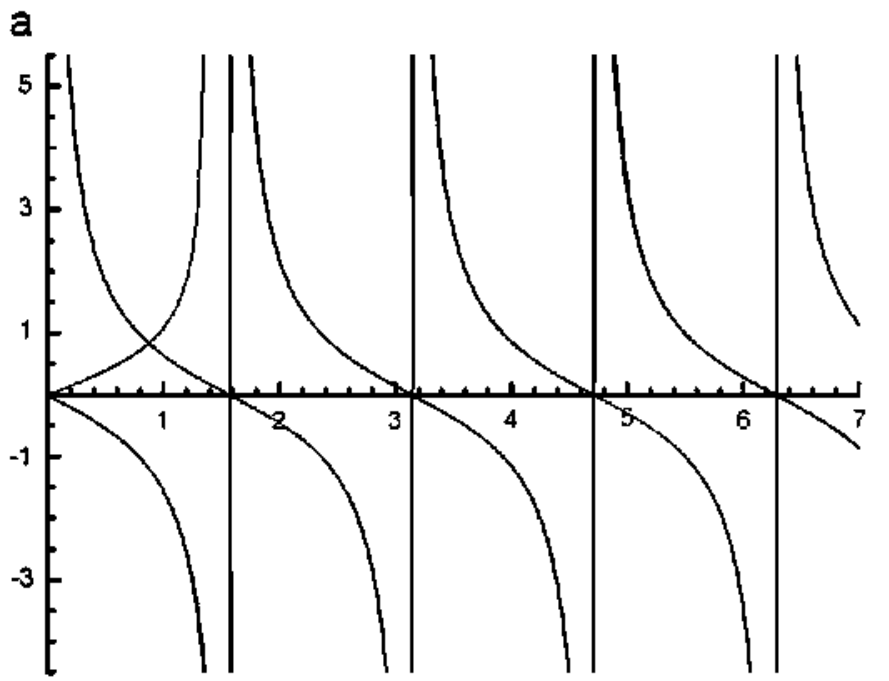

b

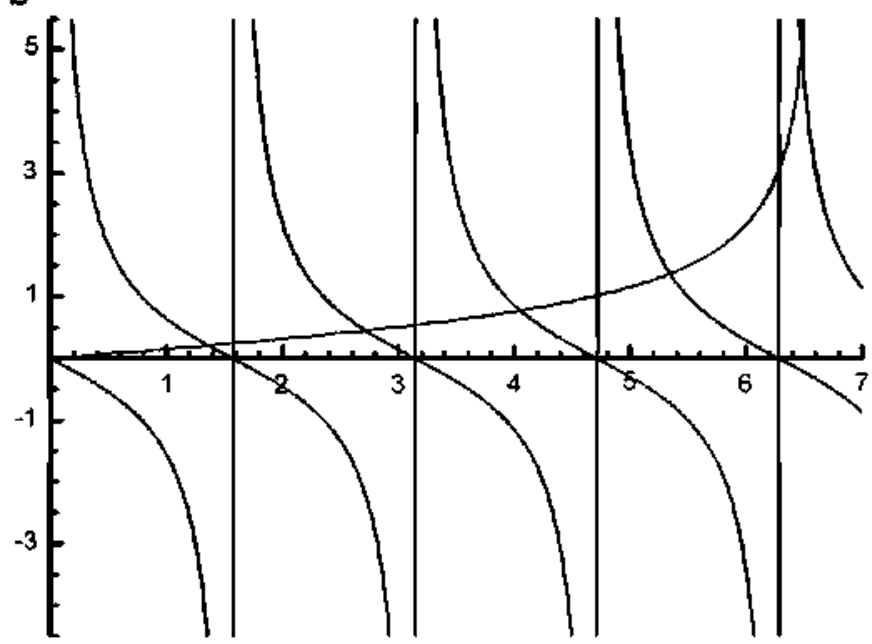

Fig. 1. The two members of the equations $-\tan \left(k_{-} c\right)=k_{z} c / \sqrt{c^{2} c^{2}-k_{2}^{2} c^{2}}$ and $\cot \left(k_{z} c\right)=k_{z} c / \sqrt{\zeta^{2} c^{2}-k_{z}^{2} c^{2}}$ vs. $k_{z} c$ for $U=0.542 \mathrm{eV}, m=0.0613$ times the electron mass; (a) $c=1.75$ and (b) $=8.5 \mathrm{~nm}$. Red curve for odd and blue for even eigenfunctions. be the well-known odd $\left(\sin \left(k_{z} z\right)\right)$ or even $\left(\cos \left(k_{z} z\right)\right)$ functions that, for $z>c$, have to be matched for function and derivative continuity with exponential functions of the type $\exp \left(-k_{z} z\right)$ and its odd or even symmetrical for $z<-c$. Calling $\zeta^{2}=\left(2 \mathrm{~m} / h^{2}\right) U$, we obtain $k_{z}^{2}=\left(2 m / h^{2}\right) E_{z}$ and $k_{z}^{2}=\zeta^{2}-k_{z}^{2}$ to verify the Schrödinger equation inside and outside the well. To verify the continuity of the logarithmic derivative, $-\tan \left(k_{z} c\right)=k_{z} c / \sqrt{\zeta^{2} c^{2}-k_{z}^{2} c^{2}}$ and $\cot \left(k_{z} c\right)=k_{z} c / \sqrt{\zeta^{2} c^{2}-k_{2}^{2} c^{2}}$ must be fulfilled for odd and even wavefunctions, respectively. These equalities are represented in Fig. 1 (with data of one of the cells actually measured). The grey curve $k_{z} c / \sqrt{\zeta^{2} c^{2}-k_{z}^{2} c^{2}}$ has a vertical asymptote for $\zeta^{2} c^{2}=k_{z}^{2} c^{2}$. This means that there are a finite number of confined states. The bigger the well dimension $c$ the further to the right in the figure is the asymptote located and more solutions (energy levels) appear, as can be seen by comparing Fig. 1 (a) and (b). At the same time, the bigger the $c$ the smaller the energy interval between energy levels (because $\Delta k_{z} c \approx \pi / 2$ and $k_{z} \wp_{-} \sqrt{E_{z}}$ ).

The eigenfunctions are characterized by their value of $k_{z} c$. Symbolically we can label them by a quantum number that will be 1 for the solution to the left, 2 for the next one, etc. Even functions correspond to the odd quantum numbers and vice versa.

Similar arguments can be used for $\alpha(x)$ and $\beta(y)$.

The eigenvalues of $\psi(x, y, z)$ are $E=E_{x}+E_{y}+E_{z}-U$ (we subtract $U$ to change the energy origin and to set it at the bottom of the host material CB). Actually, Fig. 1 (a)and (b) are related to the vertical and horizontal dimensions, respectively. There is one eigenvalue for the vertical dimension and five for each horizontal dimension, in total $5 \times 5 \times 1=25$. They are represented in Fig. 2 . States with quantum numbers $(u, w, 1)$ and $(w, u, 1)$ have same energy. This degeneracy is removed if the strain field is considered (but the splitting may be not too strong). Note that confined levels above the potential well, called virtual bound states VBS, may occur in three dimensions (never in one dimension) as described in Ref. [13]. Four levels appear as found experimentally [14] and theoretically [5] inside the potential wel].

If only one of the dimensions is very large, many closely spaced levels will appear that when the length approaches infinite will form a continuum, as occurs in quantum wires and wells.

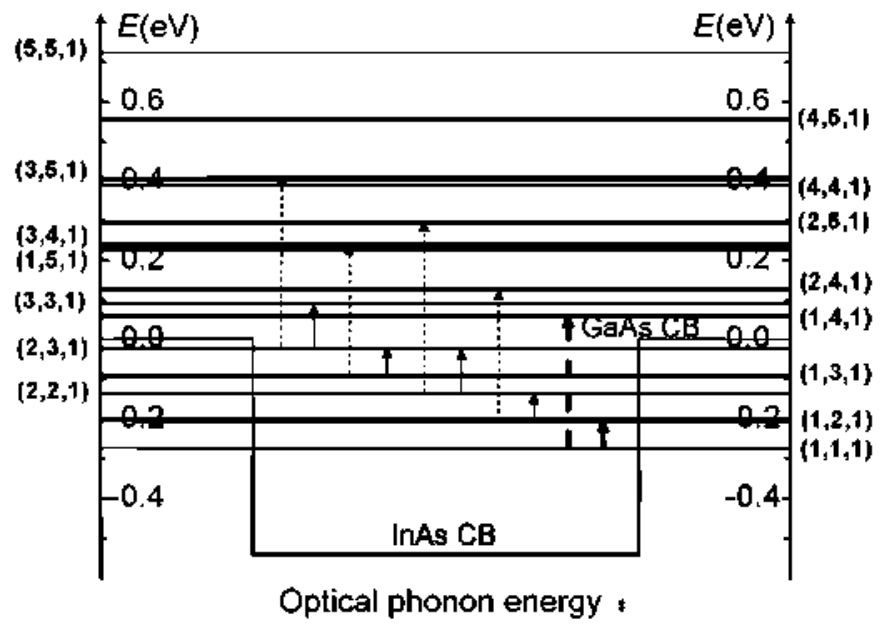

Fig. 2. Horizontal lines: confined state energy levels in an lnAs OD in GaAs; thick lines are double degenerated (besides spin degeneracy). Solid/dashed vertical arrows: strong/weak permitted optical absorptions departing from negative energy levels. Thick arrows for absorptions departing from the fundamental state. The energy of an optical phonon is drawn for comparison. 
Table 1

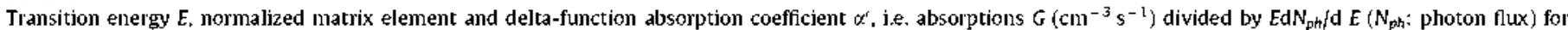

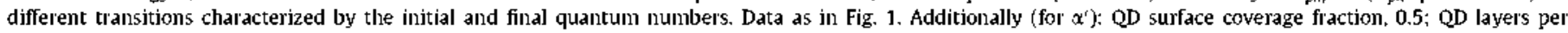
micrometer, 50; index of refraction, 3.5 .

\begin{tabular}{|c|c|c|c|c|c|c|}
\hline Transition $\left(\mathrm{ON}_{\mathbf{i}} \rightarrow \mathrm{ON}_{\mathrm{f}}\right)$ & $1 \rightarrow 2$ & $1 \rightarrow 4$ & $2 \rightarrow 3$ & $2 \rightarrow 5$ & $3 \rightarrow 4$ & $4 \rightarrow 5$ \\
\hline$E(\mathrm{eV})$ & 0.069 & 0.331 & 0.112 & 0.429 & 0.150 & 0.167 \\
\hline $\mid\left\langle\left.\psi\left|u^{*} r\right| \psi^{\prime}\right|^{2} / 8 a b\right.$ & 0.019425 & 0.00000381 & 0.024925 & 0.000255 & 0.03125 & 0.012675 \\
\hline$\alpha^{\prime}\left(\mathrm{cm}^{-1}\right)=G /(E d N p h / d E)$ & 798.75 & 0.1568125 & 1025.625 & 10.525 & 1282.5 & 521.875 \\
\hline
\end{tabular}

The levels appearing inside the QD potential well provide a ladder for electrons to escape from the QD with the absorption of phonons. The energy of an optical phonon is shown in Fig. 2 for reference.

But the method for the separation-of-variables does not solve the Schrödinger equation for the potential proposed. In effect, the box divides the space into several regions: inside the box $I,|x| \leq a$, $|y| \leq b,|z| \leq c$; in front of the 6 faces e.g. $F(100), x>a,|y| \leq b$, $|z| \leq c ;$ in front of the 12 edges, e.g. $E\left(\begin{array}{lll}1 & 1 & 0\end{array}\right), x>a, y>b,|z| \leq c$; in

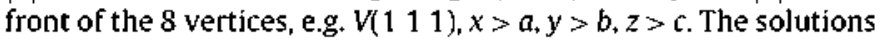
described fulfill the equation in regions $[$ and $F$ but not in region $E$ where they actually fulfill a potential $2 U$ (instead of $U$ ) or vertices where the potential is $3 U$.

It is possible to consider a perturbation potential $U_{p}$ characterized by the value 0 in region $]$ and the six regions $F,-U$ in the 12 regions $\mathrm{E}$ and $-2 U$ in the 8 regions $\mathrm{V}$. Thus, in the case of Fig. 1 the energy correction in the fundamental state $\left\langle\psi\left(1,1,1,\left|U_{p}\right| \psi(1,1,1\right.\right.$,$\rangle is -2.78 \times 10^{-8} \mathrm{eV}$. The influence in the eigenfuction is equally small, negligible for actual QDs. However it is $-0.021 \mathrm{eV}$ for cubic boxes of $3 \mathrm{~nm}$ per edge.

\section{Matrix elements}

In the one-band $\boldsymbol{k} \cdot \boldsymbol{p}$ approximation, the probability of (dipolar) photon absorption per unit of time is the same as the one obtained with the envelope function. The later is proportional (Ref. [15], vol. II, p. 899) to the element of matrix squared $\left|\left\langle\psi\left|\varepsilon^{*} r\right| \psi^{\prime}\right\rangle\right|^{2}$, where $\psi$ and $\psi^{\prime}$ are the initial and the final (normalized) eigenfunctions and $\varepsilon$ is the light polarization vector. We shall investigate the case of front ( $z$ ) illumination (common in solar cells) with plane waves in which case the polarization vector is on the $x y$ plane. By calling $\theta$, the angle of polarization with the $x$-axis (one box side) the function becomes $\mid\langle\psi| x \cos \theta+y$ $\left.\sin \theta\left|\psi^{\prime}\right\rangle\right|^{2}$, that is,

$$
\begin{aligned}
\left|\left\langle\psi\left|r^{\cdot} \varepsilon\right| \psi^{\prime}\right\rangle\right|^{2}= & \cos ^{2} \theta\left|\left\langle\psi|x| \psi^{\prime}\right\rangle\right|^{2}+\sin ^{2} \theta\left|\left\langle\psi|y| \psi^{\prime}\right\rangle\right|^{2} \\
& +2 \cos \theta \sin \theta \operatorname{Re}\left[\left\langle\psi|x| \psi^{\prime}\right\rangle\left\langle\psi|y| \psi^{\prime}\right\rangle\right]
\end{aligned}
$$

which in the case of the separation of variables is the product of three factors solely depending on $x, y$ and $z$. The $z$-depending factor is merely the internal product of two eigenfunctions, $\gamma$ and $\gamma^{\prime}$ (characterized by the value $k_{z}$ and $k_{z}$ ), that are zero unless $k_{z}=k_{z}^{\prime}$ (or they have the same quantum number $n_{z}$ ). For the term containing $\left\langle\psi|x| \psi^{\prime}\right\rangle$ the same can be said of $k_{y}$, i.e. $k_{y}=k_{y}^{\prime}$ and for the term containing $\left\langle\psi|y| \psi^{\gamma}\right\rangle, k_{x}=k_{y}^{\prime}$. Thus, either $k_{z} \neq k_{z}^{\prime}$ or $k_{y} \neq k_{y}$, but not both simultaneously. Furthermore, since the operator is an odd function, the matrix element between two odd or even functions is null. For the same reason the term containing $\left\langle\psi|x| \psi^{\prime}\right\rangle\left\langle\psi|y| \psi^{\prime}\right\rangle$ is always zero. This means that the only non-nul] terms are those differing only in either quantum number $n_{x}$ or $n_{y}$ (but not both), provided that the quantum numbers of the initial and final states are of different parity.
Note that for circular polarization the average of $\cos ^{2}$ $\theta\left|\left\langle\psi|x| \psi^{\prime}\right\rangle\right|^{2}+\sin ^{2} \theta\left|\left\langle\psi|y| \psi^{\prime}\right\rangle\right|^{2}$ is $\left|\left\langle\psi|x| \psi^{\prime}\right\rangle\right|^{2} / 2$ or $\left|\left\langle\psi|y| \psi^{\prime}\right\rangle\right|^{2} / 2$ depending on which quantum number ( $n_{x}$ or $n_{y}$ ) has changed. In the case of Fig. 1, we present some data of these transitions in Table 1 including matrix elements and absorption coefficients. Notice that only transitions between contiguous quantum numbers are important. The transitions between states that are possible, departing from energies within the GaAs bandgap are represented in Fig. 2 with an arrow (dashed if they exist, however they are negligible). Except under intense illumination, only the fundamental state is supposed to have electrons (because of the intentional doping of the $\mathrm{lB}$ region) to permit the photon absorption. We can see that no solid arrow links this state with the states that are near or inside the CB. However a weak dashed transition may be responsible for the weak two-photon effect measured in some cells [10|. Transitions between confined states and extended wavefunctions in the $C B$ are deemed to be negligible.

\section{Discussion and conclusion}

In conclusion, we have provided theoretical evidence of why the $\mathrm{IB} \rightarrow \mathrm{CB}$ transition is so elusive in the IB solar cells currently made with QDs. It is associated with the QD size and shape. Current QDs are very flat and resembles too much QWs.

A thicker height $2 c$ would lower the fundamental level approaching more to the optimal band gap distribution [1] (ideally about $0.7 \mathrm{eV}$ below the $\mathrm{CB}$ bottom). At the same time the lateral dimensions should be reduced to reduce the number of intermediate levels, reduce the tendency to thermalization and permit stronger absorption to levels above the host material $C B$. For instance, with a $\mathrm{QD}$ with $2 c=6 \mathrm{~nm}$, a typical dimension of a practical $Q D$, setting $2 a=2 b=7.28 \mathrm{~nm}$ would set the $(1,2,1)$ level $3 \mathrm{meV}$ above the $\mathrm{CB}$ and would lead to a matrix element 0.0149 times the box base area for photons of $0.248 \mathrm{eV}$. Still in this case, the separation of variables seems justified: the perturbation of the fundamental level is only of $-0.0000249 \mathrm{eV}$.

Thus what we are proposing here is to reduce the size of our QDs but more important to change the aspect ratio to become more cubic and less spread.

For a spherical potential well of the same volume of the last box the first and second levels are 0.0225 and $0.0220 \mathrm{eV}$, respectively, below those of the box (leaving the transition energy almost unchanged). This informs on the influence of the shape in the position of the levels.

\section{Acknowledgements}

This work has been supported by the IBPOWER Grant 211640 of the European Commission, by the GENESIS FV grant CSD20060004 of the Spanish programme CONSOLIDER and by the NUMANCIA grant S-0505/ENE/0310 of the Comunidad de Madrid. 


\section{References}

[1] A. Luque, A. Martí, lncreasing the efficiency of ideal solar cells by photon induced transitions at intermediate levels, Physical Review letters $78(1997)$ 5014-5017.

[2] A. Luque, A. Martí, C. Stanley, N. López, l. Cuadra, D. Zhou, A. Mc-Kee, Genera] equivalent circuit for intermediate band devices: potentials, currents and electrolumimescence. Journal of Applied Plyysics 96 (2004) 903-909.

[3] S.M. Hubbard, C.D. Cress, C.G. Bailey, R.P. Raffaelle, S.G. Bailey, D.M. Wilt Effect of strain compensation on quantum dot enhanced GaAs solar cells. Applied Physics Letters 92 (2008) 123512.

[4] R. Oshima, A. Takata, Y. Okada, Strain-compensated InAs/GaNAs quantum dots for use in high-efficiency solar cells, Applied Physics letters 93 (2008) 083111.

[5] V. Popescu, G. Bester, M.C. Hanna, A.G. Norman, A. Zunger, Theoretica] and experimental examination of the intermediate-band concept for strainbalanced $(\mathrm{ln}, \mathrm{Ga}) \mathrm{As} / \mathrm{Ga}(\mathrm{As}, \mathrm{P})$ quantum dot solar cells, Physical Review B 78 (2008) 205321

[6] S.A. Blokhin, A.V. Sakharov, A.M. Nadtochy, A.S. Pauysov, M.V. Maximov N.N. Ledentsov, A.R. Kovsh, S.S. Mikhrin, V.M. Lantratov, S.A. Mintairov N.A. Kaluzhniy, M.Z. Slivarts, AlGaAs/GaAs photovoltaic cells with an arl'ay of lnGaAs ODs, Semiconductors 43 (2009) 514-518.
[7] A. Luque, A. Marti, L. Cuadra, Thermodynamic consistency of sub-bandgap absorbing solar cellproposals. IEEE Transactions on Electron Devices 48 (2001) $2118-2124$

|8| A. Martí, A. Luque (Eds.). Next Generation Photovoltaics: High Efficiency through Full Spectrum Utilization, Institute of Physics Publishing. Bristol, 2004.

I9| K.W. Berryman, S.A. Lyon, M. Segev, Mid-infrared photoconductivity in InAs quantum dots, Applied Physics Letter's 70 (1997) 1861-1863.

[10] A. Marti, E. Antolin, C.R. Stanley, C.D. Fammer, N. Lopez, P. Diaz, E. Canovas, P.G. Linares, A. Luque, Production of photocurrent due to intermediate-toconduction-band transitions: a demonstration of a key operating principle of the intermediate-band solar cell, Physical Review Letters 97 (2006) 247701-247704

[11] S. Datta, Quantum Phenomena, Addison Wesley, Reading (Mass), 1989.

|12| D. Bimberg. M. Grundmann, N.N. ledentsov, Quantum Dot Hetrostructures, Wiley, 1999.

[13] V. Popescu, G. Bester, A. Zunger, Strain-induced localized states within the matrix continuum of self-assembled quantum dots. Applied Pliysics letter's 95 (2009) 023108.

|14| E. Cánovas, A. Martí, N. López, E. Antolín, P.G. Linares, C.D. Farmer C.R. Stanley, A. luque, Application of the photoreflectance technique to the characterization of quantum dot intemediate band materials for solar cells, Thin Solid Films 516 (2008) 6943-6947.

[15] A. Messiah, Mécanique Quantique, Dunod, Paris, 1960. 